NSF-KITP-11-173

\title{
From constrained stochastic processes to the nonlinear sigma model. Two old problems revisited
}

\author{
Franco Ferrari \\ Institute of Physics and CASA*, \\ University of Szczecin, Wielkopolska 15, \\ 70451 Szczecin, Poland \\ and \\ Kavli Institute for Theoretical Physics, \\ University of California, \\ Santa Barbara, California 93106, USA
}

\begin{abstract}
In this work a method is presented to derive the generating functional in path integral form for a system with an arbitrary number of degrees of freedom and constrained by general conditions. The method is applied to the case of the dynamics of an inextensible chain subjected to external forces. Next, the generating functional of the inextensible chain is computed assuming that the interactions are switched off. Finally, the generating functional of a two dimensional nonlinear sigma model with $\mathrm{O}(3)$ symmetry is derived exploiting its similarities with the model describing the dynamics of the inextensible chain.
\end{abstract}

\section{INTRODUCTION}

Stochastic systems in the presence of constraints are studied in connection with several physical problems [1 9]. In the absence of constraints, a very useful method in order to solve stochastic differential equations like those of Fokker-Planck consists in expressing their solution in path integral form [10-13]. Once this has been done, a rich choice of path integral techniques is available for concrete calculations. The derivation of the path integral solutions of the Fokker-Planck equations becomes however complicated when constraints are added. Various approaches have been proposed in order to cope with constrained stochastic 
differential equations [8, 9, 14 30], but the available path integral formulations for statistical systems out of equilibrium still remain awkward to be used in practical applications.

In this work a method is presented to derive for a system out of equilibrium and with an arbitrary number of degrees of freedom a path integral expression of the generating functional of the relevant correlation functions. The starting point are the Langevin equations describing the unconstrained system with the addition general constraint conditions. Roughly speaking, the strategy behind the method is to introduce fictitious degrees of freedom and white noises in order to allow the variables that should be constrained to fluctuate. It turns out that the dynamics of this extended system can be described by an enlarged set of overdamped Langevin equations, so that it is possible to construct the path integral of the related generating functional by using standard techniques. The original system of Langevin equations and of constraints is recovered in the limit in which the friction coefficients of the fictitious degrees of freedom and the widths of the fictitious noise distributions approach zero. In this limit, in the path integral of the generating functional appear functional Dirac delta functions whose role is to impose the constraints. The proposed method is tested in the case of a discrete inextensible chain, for which the generating functional of the relevant correlation functions has already been derived in path integral form in [31] and has undergone several consistency checks [32, 33]. A considerable advance with respect to [31] is the inclusion in the treatment of the external forces, that were previously missing. While with the usual approaches the path integral of the generating functional contains extra degrees of freedom (the Lagrange multipliers) $[8,9,34,35]$ or, alternatively, is complicated by functional determinants of block matrices [8, 9, 36], in the present approach the functional determinants are relatively simple and can be computed analytically.

Even with the simplest possible formulation, the treatment of system with constraints is complicated by the fact that constraints involve nonlinear functions which do not contain small or large parameters. In this situation, any approximation implies a change of the imposed conditions. For instance, within the present approach the main problem are the functional delta functions imposing the constraints. They make the computation of the generating functional extremely complicated. Usually, the constrained path integral is reduced to a gaussian one at the price of drastic simplifications [31, 37 39]. In the second part of this article it is shown in the case of the inextensible chain that it is possible to go beyond the gaussian approximation. To this purpose, the path integral of the generating functional of 
an inextensible chain is rewritten as a product of two path integrals describing two systems interacting with an auxiliary field. One of the two path integral is gaussian, while the second, which contains the Dirac delta function coming from the constraints, may be computed by performing a series expansion in powers of the auxiliary field. The series converges provided a parameter which is the analog of the Planck constant in quantum mechanics is small. An exact expression of the terms of any order appearing in the series is derived. The generating functional of these terms is computed in closed form.

The path integral of a inextensible chain in three dimensions is very similar to that of an $O(3)$ nonlinear sigma model [40] in two dimensions. For this reason, it is possible to apply also to this type of sigma models the same method used to evaluate the generating functional of the inextensible chain.

The material presented in this paper is divided as follows. In the next Section, a method for constructing the generating functional of a general constrained stochastic system is presented. As a concrete example, we apply this method to the case of a inextensible chain consisting of a set of beads connected together by massless segments of fixed length. Next, we compute the generating functional in the limit in which the chain becomes continuous. In order to do that, it is necessary to perform a path integral that is complicated by the presence of a functional Dirac delta function related to the constraints. The path integration is simplified by the introduction of suitable auxiliary fields. At the end, a nonperturbative

expression of the generating functional is obtained. Finally, a similar method is exploited to compute the generating functional of the correlation functions of a two dimensional nonlinear sigma model with $O(3)$ group of symmetry.

\section{CONSTRAINED STOCHASTIC PROCESSES}

Let $\{\boldsymbol{R}\}=\boldsymbol{R}_{1}(t), \ldots, \boldsymbol{R}_{N}(t)$ denote the radius vectors of a set of $N$ particles in three dimensions. The positions of the particles are constrained by the conditions:

$$
C_{\alpha}(\{\boldsymbol{R}\})=0 \quad \alpha=1, \ldots, M<3 N
$$

Their motion is subjected to the forces $\boldsymbol{F}_{i}=\boldsymbol{f}+\boldsymbol{\nu}_{i}$ for $i=1, \ldots, N$. Apart from a rescaling by a dimensional constant, namely the inverse of the friction coefficient, the $\boldsymbol{f}_{i}=\boldsymbol{f}_{i}(\{\boldsymbol{R}\})$ are external forces, while the $\boldsymbol{\nu}_{i}$ 's represent random forces with gaussian distribution, whose 
properties are determined by the following correlation functions:

$$
\begin{aligned}
\left\langle\boldsymbol{\nu}_{i}(t)\right\rangle & =0 \\
\left\langle\nu_{i, a}(t) \nu_{j, b}\left(t^{\prime}\right)\right\rangle & =2 D \delta_{i j} \delta_{a b} \delta\left(t-t^{\prime}\right)
\end{aligned}
$$

Here $a, b=1,2,3$ label the spatial components and $D$ characterizes the width of the noise distribution. Assuming that the particles are fluctuating in a viscous solution, their motion will be described by the overdamped stochastic equation:

$$
\dot{\boldsymbol{R}}_{i}=\boldsymbol{f}_{i}+\boldsymbol{\nu}_{i} \quad i=1, \ldots, N
$$

to which one has to add the conditions (11). Following a standard notation, in Eq. (41) it has been put $\dot{\boldsymbol{R}}_{i}(t)=\frac{\partial \boldsymbol{R}_{i}(t)}{\partial t}$. Before passing from Eqs. (11) and (44) to a path integral formulation of the generating functional of the correlation functions

$$
G_{i_{1}, \ldots, i_{n}, a_{1}, \ldots, a_{n}}\left(t_{1}, \ldots, t_{n}\right)=\left\langle R_{i_{1}, a_{1}}\left(t_{1}\right), \ldots, R_{i_{n}, a_{n}}\left(t_{n}\right)\right\rangle
$$

we briefly present a strategy explained in [41] in order to construct the generating functional in the simple case of the Langevin equation of a particle moving along the $x$-axis:

$$
m \ddot{x}+\eta \dot{x}-f=\nu
$$

with $\eta$ being the friction coefficient. Due to the inertial term, Eq. (66) is of second order in time. The idea of [41] is to transform it in a system of two equations of the first order by introducing the new variable

$$
v=\dot{x}
$$

In the enlarged two dimensional space $(x, v)$, Eq. (7) looks like a constraint which can be imposed á la Parisi-Wu by adding a fictitious random force $\tilde{\nu}$ with a gaussian noise distribution characterized by the standard deviation $\tilde{D}$. In this way, one obtains from Eq. (6) two overdamped Langevin equations:

$$
\begin{aligned}
m \dot{v}+\eta v-f & =\nu \\
\dot{x}-v & =\tilde{\nu}
\end{aligned}
$$

Starting from the above system of equations, the construction of the generating functional of the correlation functions is straightforward. Unfortunately, this method for fixing the 
constraints cannot be applied to our case without changes because of two relevant differences. First, the constraint (7) is imposed on a new variable, the velocity $v$, that was not present in the original Langevin equation (6). For this reason, there is since the beginning a clear distinction between the physical and redundant degrees of freedom. In principle, it is possible to extract also from Eqs. (11) and (44) a set of physical variables $u_{1}, \ldots, u_{3 N-M}$ such that $C_{\alpha}\left(\boldsymbol{R}_{1}\left(u_{1}, \ldots, u_{3 N-M}\right), \ldots, \boldsymbol{R}_{N}\left(u_{1}, \ldots, u_{3 N-M}\right)\right)=0$ for $\alpha=1, \ldots, M$. The velocities $v_{1}, \ldots, v_{M}$ could then be identified with the spurious degrees of freedom living in the subspace of $\mathbb{R}^{3 N}$ which is orthogonal to that spanned by the $u$ 's. The major difficulty of this program is that, in order to determine the variables $u_{1}, \ldots, u_{3 N-M}$, it is necessary to solve the relations (11). This task is in general not feasible. On the second hand, Eq. (7) is already in the form of an overdamped Langevin equation apart from the presence of the noise, while Eqs. (1) are not. To eliminate these differences, we introduce the new degrees of freedom $\xi_{\alpha}$ and new random forces $\tilde{\nu}_{\alpha}, \alpha=1, \ldots, M$, characterized by the correlation functions:

$$
\begin{aligned}
\left\langle\tilde{\nu}_{\alpha}(t)\right\rangle & =0 \\
\left\langle\tilde{\nu}_{\alpha}(t) \tilde{\nu}_{\beta}\left(t^{\prime}\right)\right\rangle & =2 \tilde{D}_{\alpha} \delta_{\alpha \beta} \delta\left(t-t^{\prime}\right)
\end{aligned}
$$

At this point, we replace Eqs. (1) and (4) with the following set of Langevin equations:

$$
\begin{aligned}
\dot{\boldsymbol{R}}_{i} & =\boldsymbol{f}_{i}+\boldsymbol{\nu}_{i} \\
\eta_{\alpha} \dot{\xi}_{\alpha}+C_{\alpha}(\{\boldsymbol{R}\}) & =\tilde{\nu}_{\alpha}
\end{aligned}
$$

In the equilibrium limit $\tilde{D}_{\alpha} \rightarrow 0$ and by requiring that the friction constants $\eta_{\alpha}$ vanish identically, the Langevin equations (13) reduce to the original constraints (1). The main advantage of having enlarged in this way the space of variables is that now it is possible to apply the standard procedure for constructing the expression of the generating functional. First of all, we introduce two sets of external currents $\boldsymbol{J}_{i}, i=1, \ldots, N$ and $\tilde{j}_{\alpha}, \alpha=1, \ldots, M$. The generating functional may be written as follows:

$$
\begin{aligned}
Z[\boldsymbol{J}, \tilde{j}] & =\left[\prod_{i=1}^{N} \prod_{\alpha=1}^{M} \lim _{\tilde{D}_{\alpha} \rightarrow 0} \lim _{\eta_{\alpha} \rightarrow 0} \int \mathcal{D} \boldsymbol{\nu}_{i} \mathcal{D} \tilde{\nu}_{\alpha}\right] \exp \left\{-\int_{0}^{t_{f}} d t\left(\sum_{i=1}^{N} \frac{\boldsymbol{\nu}_{i}^{2}}{4 D}+\sum_{\alpha=1}^{M} \frac{\tilde{\nu}_{\alpha}^{2}}{4 \tilde{D}_{\alpha}}\right)\right\} \\
& \times \exp \left\{-\int_{0}^{t_{f}} d t\left[\sum_{\alpha=1}^{M} \xi_{\alpha} \tilde{j}_{\alpha}+\imath \sum_{i=2}^{N} \boldsymbol{R}_{i} \cdot\left(\boldsymbol{J}_{i}-\boldsymbol{J}_{i-1}\right)\right]\right\}
\end{aligned}
$$

In the above equation the quantity $t_{f}$ denotes the upper limit of the time interval in which the evolution of the system is followed. We stress the fact that the current term for the 
radius vectors $\imath \int_{0}^{t_{f}} d t \sum_{i=2}^{N} \boldsymbol{R}_{i} \cdot\left(\boldsymbol{J}_{i}-\boldsymbol{J}_{i-1}\right)$, with $\imath$ denoting the imaginary unit, contains the discrete derivative of the external currents $\boldsymbol{J}_{i}$ and not the current itself. This definition of the current term is a just matter of future convenience and does not reduce the generality of our treatment. Let us also note that in Eq. (14) the $\xi_{\alpha}$ 's and the $\boldsymbol{R}_{i}$ 's represent respectively the solutions of Eqs. (13) and (12) and thus they depend on the noises $\tilde{\nu}_{\alpha}$ and $\boldsymbol{\nu}_{i}$. Moreover, the $\boldsymbol{R}_{i}(t)$ 's satisfy the time boundary conditions:

$$
\boldsymbol{R}_{i}(0)=\boldsymbol{R}_{0, i} \quad \boldsymbol{R}_{i}\left(t_{f}\right)=\boldsymbol{R}_{f, i}
$$

Here the $\boldsymbol{R}_{0, i}$ 's denote the fixed initial conformations of the chain, while the $\boldsymbol{R}_{f, i}$ 's describe the conformation at the final time $t=t_{f}$. Next, we perform in Eq. (14) the change of variables $\boldsymbol{\nu}_{i}, \tilde{\nu}_{\alpha} \longrightarrow \boldsymbol{R}_{i}, \xi_{\alpha}$ :

$$
\begin{aligned}
& Z[\boldsymbol{J}, \tilde{j}]=\left[\prod_{i=1}^{N} \prod_{\alpha=1}^{M} \lim _{\tilde{D}_{\alpha} \rightarrow 0} \lim _{\eta_{\alpha} \rightarrow 0} \int_{\boldsymbol{R}_{i}(0)=\boldsymbol{R}_{0, i}}^{\boldsymbol{R}_{i}(f)=\boldsymbol{R}_{f, i}} \mathcal{D} \boldsymbol{R}_{i} \mathcal{D} \xi_{\alpha}\right] J a c \exp \left\{-\sum_{i=1}^{N} \int_{0}^{t_{f}} d t \frac{\left(\dot{\boldsymbol{R}}_{i}-\boldsymbol{f}\right)^{2}}{4 D}\right\} \\
& \times \exp \left\{-\sum_{\alpha=1}^{M} \int_{0}^{t_{f}} \frac{\left(\eta \dot{\xi}_{\alpha}+C_{\alpha}(\{\boldsymbol{R}\})\right)^{2}}{4 \tilde{D}_{\alpha}}\right\} \exp \left\{-\int_{0}^{t_{f}} d t\left[\sum_{\alpha=1}^{M} \xi_{\alpha} \tilde{j}_{\alpha}+\imath \sum_{i=2}^{N} \boldsymbol{R}_{i} \cdot\left(\boldsymbol{J}_{i}-\boldsymbol{J}_{i-1}\right)\right]\right\}
\end{aligned}
$$

where $J a c$ is the determinant of the transformation. In principle, Jac is the determinant of a complicated block matrix:

$$
J a c=\operatorname{det}\left|\begin{array}{cc}
\frac{\delta \nu_{i, a}}{\delta R_{j, b}} & \frac{\delta \nu_{i, a}}{\delta \xi_{\beta}} \\
\frac{\delta \tilde{\nu}_{\alpha}}{\delta R_{j, b}} & \frac{\delta \tilde{\nu}_{\alpha}}{\delta \xi_{\beta}}
\end{array}\right|
$$

The determinant in the right hand side of Eq. (17) can however be simplified by noticing that Eqs. (12) establish relationships only between the $\boldsymbol{\nu}_{i}$ 's and the $\boldsymbol{R}_{j}$ 's. As a consequence, it turns out that $\frac{\delta \nu_{i, a}}{\delta \xi_{\beta}}=0$ for $i=1, \ldots, N, a=1,2,3$ and $\beta=1, \ldots, M$. For this reason, $J a c$ reduces to a product of two determinants:

$$
J a c=\operatorname{det}\left|\frac{\delta \nu_{i, a}}{\delta R_{j, b}}\right| \operatorname{det}\left|\frac{\delta \tilde{\nu}_{\alpha}}{\delta \xi_{\beta}}\right|
$$

A simple way to prove the above identity is to eliminate first the $\boldsymbol{\nu}_{i}$ 's in the functional integral (14) using Eqs. (12). Clearly, from those equations it turns out that $\boldsymbol{\nu}_{i}(\boldsymbol{R})=\dot{\boldsymbol{R}}_{i}-\boldsymbol{f}_{i}$. The jacobian of this transformation is $\operatorname{det}\left|\frac{\delta \nu_{i, a}}{\delta R_{j, b}}\right|$. After substituting everywhere in (14) the $\boldsymbol{\nu}_{i}$ 's with their new expression in terms of the $\boldsymbol{R}_{i}$ 's, we may eliminate also the auxiliary noises $\tilde{\nu}_{\alpha}$ exploiting Eqs. (13). As a result, the $\tilde{\nu}_{\alpha}$ become functions of the new variables $\boldsymbol{R}_{i}$ 's and $\xi_{\alpha}$ 's. The jacobian determinant of this second transformation is $\operatorname{det}\left|\frac{\delta \tilde{\nu}_{\alpha}}{\delta \xi_{\beta}}\right|$. This completes 
our proof. Alternatively, Eq. (18) may be easily checked by noticing that in the calculation of the full determinant in the right hand side of Eq. (17) each element of the matrix $\frac{\delta \tilde{\nu}_{\alpha}}{\delta R_{j, b}}$ is necessarily multiplied by an element of the matrix $\frac{\delta \nu_{i, a}}{\delta \xi_{\beta}}$ which is equal to zero.

Explicitly, the determinants in Eq. (18) read as follows:

$$
\operatorname{det}\left|\frac{\delta \nu_{i, a}(t)}{\delta R_{j, b}\left(t^{\prime}\right)}\right|=\operatorname{det}\left|\left(\delta_{i j} \delta_{a b} \frac{\partial}{\partial t}-\frac{\partial f_{i, a}(t)}{\partial R_{j, b}(t)}\right) \delta\left(t-t^{\prime}\right)\right|
$$

with $f_{i, a}(t)=f_{i, a}\left(\boldsymbol{R}_{1}(t), \ldots, \boldsymbol{R}_{N}(t)\right)$ and

$$
\operatorname{det}\left|\frac{\delta \tilde{\nu}_{\alpha}(t)}{\delta \xi_{\beta}\left(t^{\prime}\right)}\right|=\eta_{\alpha} \frac{\partial}{\partial t} \delta\left(t-t^{\prime}\right)
$$

The only relevant determinant is that in Eq. (19). Determinants of this type can be evaluated in closed form. We give here only the result of the calculation referring the interested reader for all details to standard books in this subject like for instance Ref. [10]:

$$
\operatorname{det}\left|\left(\delta_{i j} \delta_{a b} \frac{\partial}{\partial t}-\frac{\partial f_{i, a}(t)}{\partial R_{j, b}(t)}\right) \delta\left(t-t^{\prime}\right)\right|=\exp \left[-\frac{1}{4} \int_{0}^{t_{f}} d t \sum_{i=1}^{N} \sum_{a=1}^{3} \frac{\partial f_{i, a}(t)}{\partial R_{i, a}(t)}\right]
$$

Putting the expression of the jacobian just computed in Eq. (16) and taking the limit $\eta_{\alpha} \rightarrow 0$, we obtain:

$$
\begin{aligned}
Z[\boldsymbol{J}] & =\left[\prod_{i=1}^{N} \int_{\boldsymbol{R}_{i}(0)=\boldsymbol{R}_{0, i}}^{\boldsymbol{R}_{i}(f)=\boldsymbol{R}_{f, i}} \mathcal{D} \boldsymbol{R}_{i}\right] \exp \left\{-\frac{1}{4 D} \sum_{i=1}^{N} \int_{0}^{t_{f}} d t\left(\dot{\boldsymbol{R}}_{i}-\boldsymbol{f}_{i}\right)^{2}\right\} \\
& \times \exp \left[-\frac{1}{4} \int_{0}^{t_{f}} d t \sum_{i=1}^{N} \sum_{a=1}^{3} \frac{\partial f_{i, a}(t)}{\partial R_{i, a}(t)}\right] \exp \left\{-\int_{0}^{t_{f}} d t\left[\imath \sum_{i=2}^{N} \boldsymbol{R}_{i} \cdot\left(\boldsymbol{J}_{i}-\boldsymbol{J}_{i-1}\right)\right]\right\} \\
& \times\left[\prod_{\alpha=1}^{M} \lim _{\tilde{D}_{\alpha} \rightarrow 0}\right] \exp \left[-\sum_{\alpha=1}^{M} \frac{1}{4 \tilde{D}_{\alpha}} \int_{0}^{t_{f}} d t C_{\alpha}^{2}(\{\boldsymbol{R}\})\right]
\end{aligned}
$$

The $\xi_{\alpha}$ 's decouple from the other degrees of freedom in the limit $\eta_{\alpha} \rightarrow 0$ and have been already integrated out in Eq. (22). It remains to perform the limit $\tilde{D}_{\alpha} \rightarrow 0$. To this purpose it is possible to apply the formula [31, 33]:

$$
\lim _{\sigma \rightarrow 0} e^{-\frac{1}{\sigma} \int_{0}^{t_{f}} f^{2}(\{\boldsymbol{R}\})}=\delta(f(\{\boldsymbol{R}\}))
$$

As a result, Eq. (22) becomes:

$$
\begin{aligned}
Z[\boldsymbol{J}] & =\left[\prod_{i=1}^{N} \int_{\boldsymbol{R}_{i}(0)=\boldsymbol{R}_{0, i}}^{\boldsymbol{R}_{i}(f)=\boldsymbol{R}_{f, i}} \mathcal{D} \boldsymbol{R}_{i}\right] \exp \left\{-\frac{1}{4 D} \sum_{i=1}^{N} \int_{0}^{t_{f}} d t\left(\dot{\boldsymbol{R}}_{i}^{2}+\boldsymbol{f}_{i}^{2}\right)\right\} \\
& \times \exp \left[-\frac{1}{4} \int_{0}^{t_{f}} d t \sum_{i=1}^{N} \sum_{a=1}^{3} \frac{\partial f_{i, a}(t)}{\partial R_{i, a}(t)}\right] \exp \left\{-\int_{0}^{t_{f}} d t\left[\imath \sum_{i=2}^{N} \boldsymbol{R}_{i} \cdot\left(\boldsymbol{J}_{i}-\boldsymbol{J}_{i-1}\right)\right]\right\} \\
& \times\left[\prod_{\alpha=1}^{M} \delta\left(C_{\alpha}(\{\boldsymbol{R}\})\right)\right]
\end{aligned}
$$


This is the desired expression of the generating functional $Z[\boldsymbol{J}]$. In writing the above formula we have have assumed that the forces $\boldsymbol{f}_{i}$ are conservative, so that it is possible to use the identity $\int_{0}^{t_{f}} d t\left(\dot{\boldsymbol{R}}_{i}-\boldsymbol{f}_{i}\right)^{2}=\int_{0}^{t_{f}}\left(\dot{\boldsymbol{R}}_{i}^{2}+\boldsymbol{f}_{i}^{2}\right)$ which is valid up to a term which is a total derivative.

\section{THE EXAMPLE OF AN INEXTENSIBLE CHAIN}

As an example of the method for imposing the constraints explained in the previous Section, we consider here the generating functional $Z^{i . c .}[\boldsymbol{J}]$ describing the dynamics of an inextensible chain fluctuating in a viscous media. The chain is a discrete mechanical system consisting of $N$ beads of mass $m$ connected together by $N-1$ massless segments of fixed length $a$. The total length of the chain is $L=N a$ and its total mass is $M=N m$. For this particular example the constraints (1) take the form:

$$
C_{\alpha}(\{\boldsymbol{R}\})=\left|\boldsymbol{R}_{\alpha}-\boldsymbol{R}_{\alpha-1}\right|^{2}-a^{2}=0 \quad \alpha=2, \ldots, N
$$

The direct application of Eq. (24) to the inextensible chain gives as a result the generating functional:

$$
\begin{aligned}
Z^{i . c .}[\boldsymbol{J}] & =\left[\prod_{i=1}^{N} \int_{\boldsymbol{R}_{i}(0)=\boldsymbol{R}_{0, i}}^{\boldsymbol{R}_{i}(f)=\boldsymbol{R}_{f, i}} \mathcal{D} \boldsymbol{R}_{i}\right] \exp \left\{-\frac{1}{4 D} \sum_{i=1}^{N} \int_{0}^{t_{f}} d t\left(\dot{\boldsymbol{R}}_{i}^{2}-\boldsymbol{f}_{i}^{2}\right)\right\} \\
& \times \exp \left[-\frac{1}{4} \int_{0}^{t_{f}} d t \sum_{i=1}^{N} \sum_{a=1}^{3} \frac{\partial f_{i, a}(t)}{\partial R_{i, a}(t)}\right] \exp \left\{-\int_{0}^{t_{f}} d t\left[\imath \sum_{i=2}^{N} \boldsymbol{R}_{i} \cdot\left(\boldsymbol{J}_{i}-\boldsymbol{J}_{i-1}\right)\right]\right\} \\
& \times\left[\prod_{\alpha=2}^{N} \delta\left(\left|\boldsymbol{R}_{\alpha}-\boldsymbol{R}_{\alpha-1}\right|^{2}-a^{2}\right)\right]
\end{aligned}
$$

In the following all external forces will be switched off, i. e. $\boldsymbol{f}_{i}=0$. We will now consider the continuous limit of $Z^{\text {i.c. }}[\boldsymbol{J}]$ :

$$
a \rightarrow 0 \quad N \rightarrow \infty \quad N a=L=\text { const. }
$$

This limit can be performed using the prescription of Ref. [31]. The result is the partition function

$$
\mathcal{Z}[\boldsymbol{J}]=\lim _{a \rightarrow 0, N \rightarrow \infty, N a=L} Z^{i . c .}[\boldsymbol{J}]
$$

of what has been called the generalized nonlinear sigma model in Ref. [31]:

$$
\mathcal{Z}[\boldsymbol{J}]=\int_{\boldsymbol{R}(0, s)=\boldsymbol{R}_{0}(s)}^{\boldsymbol{R}\left(t_{f}, s\right)=\boldsymbol{R}_{f}(s)} \mathcal{D} \boldsymbol{R}(t, s) e^{-\int_{0}^{t_{f}} d t \int_{0}^{L}\left(c \dot{\boldsymbol{R}}^{2}(t, s)+\boldsymbol{R}(t, s) \cdot \boldsymbol{J}^{\prime}(t, s)\right)} \delta\left(\left|\boldsymbol{R}^{\prime}(t, s)\right|^{2}-1\right)
$$


In the above equation $\boldsymbol{R}_{0}(s)$ and $\boldsymbol{R}_{f}(s)$ represent the continuous version of the discrete initial and final conformations of the chain given in Eq. (15). Moreover, $s$ is the arc-length on the chain and $\boldsymbol{R}^{\prime}(t, s)=\frac{\partial R(t, s)}{\partial s}$. Finally,

$$
c=\frac{1}{2 k_{B} T \tau} \frac{M}{2 L}
$$

with $k_{B}$ being the Boltzmann constant. The parameter $c$ contains physical constants like the temperature of the viscous medium $T$ and the relaxation time of the infinitesimal beads $\tau$. In the frame of the duality between statistical physics and quantum mechanics, the quantity $2 k_{B} T \tau$ is the analog of the Planck constant [42]. It describes the uncertainties in determining the positions and the momenta of the beads because of the collisions with the molecules of the surrounding medium.

Up to now, the generating functional $\mathcal{Z}[\boldsymbol{J}]$ has been computed in the semiclassical approximation [31] or it has been linearized using a variational method [33]. In the rest of this Section an attempt to go beyond these approximations will be presented. In order to avoid as much as possible complications with the boundary conditions, we will suppose that the chain is ring-shaped. Accordingly, throughout the rest of this Section all the fields will satisfy periodic boundary conditions of the type:

$$
\boldsymbol{R}(t, s+L)=\boldsymbol{R}(t, s)
$$

The functional Dirac delta function imposing the constraints in Eq. (29) may be treated by introducing the new field $\boldsymbol{r}(t, s)$ and rewriting $\mathcal{Z}[\boldsymbol{J}]$ as follows:

$$
\begin{aligned}
\mathcal{Z}[\boldsymbol{J}] & =\int_{\boldsymbol{R}(0, s)=\boldsymbol{R}_{0}(s)}^{\boldsymbol{R}\left(t_{f}, s\right)=\boldsymbol{R}_{f}(s)} \mathcal{D} \boldsymbol{R}(t, s) \int_{\boldsymbol{r}(0, s)=\boldsymbol{R}_{0}^{\prime}(s)}^{\boldsymbol{r}\left(t_{f}, s\right)=\boldsymbol{R}_{f}^{\prime}(s)} \mathcal{D} \boldsymbol{r}(t, s) e^{-\int_{0}^{t_{f}} d t \int_{0}^{L}\left(c \dot{\boldsymbol{R}}^{2}(t, s)+\boldsymbol{R}(t, s) \cdot \boldsymbol{J}^{\prime}(t, s)\right)} \\
& \times \delta\left(\boldsymbol{r}(t, s)-\boldsymbol{R}^{\prime}(t, s)\right) \delta\left(\boldsymbol{r}^{2}(t, s)-1\right)
\end{aligned}
$$

When $t=0$ and $t=t_{f}, \boldsymbol{r}(t, s)$ obeys respectively the boundary conditions:

$$
\boldsymbol{r}(0, s)=\boldsymbol{R}_{i}^{\prime}(s) \quad \text { and } \quad \boldsymbol{r}\left(t_{f}, s\right)=\boldsymbol{R}_{f}^{\prime}(s)
$$

At this point we express the first delta function appearing in the right hand side of Eq. (32) using the Fourier representation:

$$
\delta\left(\boldsymbol{r}-\boldsymbol{R}^{\prime}\right)=\int \mathcal{D} \boldsymbol{k} e^{\imath \int_{0}^{t_{f}} d t \int_{0}^{L} d s \boldsymbol{k} \cdot\left(\boldsymbol{r}-\boldsymbol{R}^{\prime}\right)}
$$


The new Fourier variable $\boldsymbol{k}=\boldsymbol{k}(t, s)$ must be free to vary in the interval $(-\infty,+\infty)$ over the whole domain $[0, L] \times\left(0, t_{f}\right)$ of $s$ and $t$ in order to impose the relation $\boldsymbol{r}(t, s)=\boldsymbol{R}^{\prime}(t, s)$. When $t=0$ and $t=t_{f}$, instead, the values of $\boldsymbol{r}(0, s)$ and $\boldsymbol{r}\left(t_{f}, s\right)$ have been already fixed in the generating functional of Eq. (32) to be those of Eq. (33). For this reason, at the instants $t=0$ and $t=t_{f}$ we may chose for $\boldsymbol{k}(t, s)$ Dirichlet boundary conditions, i. e.

$$
\boldsymbol{k}(0, s)=\boldsymbol{k}\left(t_{f}, s\right)=0
$$

Applying Eq. (34) in Eq. (32), it is possible to split $\mathcal{Z}[\boldsymbol{J}]$ into a path integral over $\boldsymbol{k}(t, s)$ of the product of two different contributions:

$$
\mathcal{Z}[\boldsymbol{J}]=\int \mathcal{D} \boldsymbol{k} \mathcal{Z}_{1}[\boldsymbol{k}, \boldsymbol{J}] \mathcal{Z}_{2}[\boldsymbol{k}]
$$

where

$$
\mathcal{Z}_{1}[\boldsymbol{k}, \boldsymbol{J}]=\int_{\boldsymbol{R}(0, s)=\boldsymbol{R}_{0}(s)}^{\boldsymbol{R}\left(t_{f}, s\right)=\boldsymbol{R}_{f}(s)} \mathcal{D} \boldsymbol{R} \exp \left\{-\int_{0}^{t_{f}} d t \int_{0}^{L} d s\left[c \dot{\boldsymbol{R}}^{2}+\imath\left(\boldsymbol{J}^{\prime}+\boldsymbol{k}^{\prime}\right) \cdot \boldsymbol{R}\right]\right\}
$$

and

$$
\mathcal{Z}_{2}[\boldsymbol{k}]=\int_{\boldsymbol{r}(0, s)=\boldsymbol{R}_{0}^{\prime}(s)}^{\boldsymbol{r}\left(t_{f}, s\right)=\boldsymbol{R}_{f}^{\prime}(s)} \mathcal{D} \boldsymbol{r} e^{+\imath \int_{0}^{t_{f}} d t \int_{0}^{L} d s \boldsymbol{k} \cdot \boldsymbol{r}} \delta\left(\boldsymbol{r}^{2}-1\right)
$$

As we see, the integration over $\boldsymbol{R}(t, s)$ is completely disentangled from that over $\boldsymbol{r}(t, s)$, but both fields interact with $\boldsymbol{k}(t, s)$.

First, the functional $\mathcal{Z}_{1}[\boldsymbol{k}, \boldsymbol{J}]$ will be computed. To this purpose, it is convenient to perform the change of variables:

$$
\boldsymbol{R}=\boldsymbol{R}_{c l}+\delta \boldsymbol{R}
$$

Here $\boldsymbol{R}_{c l}$ is a solution of the free classical equation of motion $\ddot{\boldsymbol{R}}_{c l}=0$ and satisfies the same boundary conditions as $\boldsymbol{R}$. Consequently, the fluctuation $\delta \boldsymbol{R}$ obeys the Dirichlet boundary conditions:

$$
\delta \boldsymbol{R}\left(t_{f}, s\right)=\delta \boldsymbol{R}(0, s)=0
$$

Moreover, both $\boldsymbol{R}_{c l}(t, s)$ and $\delta \boldsymbol{R}$ are periodic in $s$. After a gaussian integration over $\delta \boldsymbol{R}$, we obtain:

$\mathcal{Z}_{1}[\boldsymbol{k}, \boldsymbol{J}]=e^{-S_{c l}(\boldsymbol{k}, \boldsymbol{J})} \exp \left\{-\frac{1}{4} \int_{0}^{t_{f}} d t d t^{\prime} \int_{0}^{L} d s G\left(t, t^{\prime}\right)\left(\boldsymbol{J}^{\prime}(t, s)+\boldsymbol{k}^{\prime}(t, s)\right) \cdot\left(\boldsymbol{J}^{\prime}\left(t^{\prime}, s\right)+\boldsymbol{k}^{\prime}\left(t^{\prime}, s\right)\right)\right\}$

where

$$
S_{c l}(\boldsymbol{k}, \boldsymbol{J})=\int_{0}^{t_{f}} d t \int_{0}^{L} d s\left[c \dot{\boldsymbol{R}}_{c l}^{2}+\imath\left(\boldsymbol{J}^{\prime}+\boldsymbol{k}^{\prime}\right) \cdot \boldsymbol{R}_{c l}\right]
$$


while $G\left(t, t^{\prime}\right)$ is the Green function satisfying the equation:

$$
2 c \frac{\partial^{2} G\left(t, t^{\prime}\right)}{\partial t^{2}}=\delta\left(t-t^{\prime}\right)
$$

and Dirichlet boundary conditions. Explicitly:

$$
G\left(t, t^{\prime}\right)=\frac{1}{2 c t_{f}}\left[\frac{\left(t+t^{\prime}\right)}{2}-\frac{\left|t-t^{\prime}\right|}{2}\right]\left[\frac{\left(t+t^{\prime}\right)}{2}+\frac{\left|t-t^{\prime}\right|}{2}-t_{f}\right]
$$

Next, we consider the functional $\mathcal{Z}_{2}[\boldsymbol{k}]$. This quantity closely resembles the Fourier transform of the probability distribution of a freely jointed chain appearing in the statistical mechanics of polymers. It may be computed exactly in a similar way using a discretization procedure. We have already seen how to pass from the continuous interval $[0, L]$ to its discrete approximation in the case of the arc-length $s$, see e. g. Eq. (27). Analogously, we discretize the time interval $\left[0, t_{f}\right]$ by replacing it with a lattice of $\mathcal{M}$ sites and link length $b$. The continuous limit is performed by requiring that:

$$
b \rightarrow 0 \quad \mathcal{M} \rightarrow+\infty \quad \mathcal{M} b=t_{f}
$$

Ignoring for the moment the nontrivial time boundary conditions of $\boldsymbol{r}(t, s)$, a straightforward calculation shows that $\mathcal{Z}_{2}[\boldsymbol{k}]$ is given by:

$$
\mathcal{Z}_{2}[\boldsymbol{k}]=\lim _{\substack{a \rightarrow 0, N \rightarrow+\infty, N a=L \\ b \rightarrow 0, \mathcal{M} \rightarrow+\infty, \mathcal{M} b=t_{f}}} \prod_{n=1}^{N} \prod_{m=1}^{\mathcal{M}} \frac{\sin \left(a b\left|\boldsymbol{k}_{n m}\right|\right)}{a b\left|\boldsymbol{k}_{n m}\right|}
$$

Here $\boldsymbol{k}_{n m}$ is a shorthand notation for $\boldsymbol{k}\left(t_{m}, s_{n}\right)$. Apart from the boundary conditions, the problem with Eq. (46) is the evaluation of the continuous limit of its right hand side. This is a nontrivial task. The naive prescription used in the statistical mechanics of polymers [14, 43], i. e.

$$
\frac{\sin \left(a b\left|\boldsymbol{k}_{n m}\right|\right)}{a b\left|\boldsymbol{k}_{n m}\right|} \sim 1-\left(a b\left|\boldsymbol{k}_{n m}\right|\right)^{2} \sim e^{-\left(a b\left|\boldsymbol{k}_{n m}\right|\right)^{2}}
$$

fails to provide the correct result. For that reason, in order to evaluate $\mathcal{Z}_{2}[\boldsymbol{k}]$, we expand

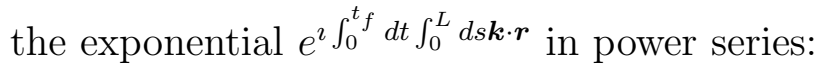

$$
\mathcal{Z}_{2}[\boldsymbol{k}]=\sum_{\sigma=0}^{+\infty} \frac{(-\imath)^{2 \sigma}}{2 \sigma !} I_{2 \sigma}
$$

where

$$
I_{2 \sigma}=\int_{\boldsymbol{r}(0, s)=\boldsymbol{R}_{0}^{\prime}(s)}^{\boldsymbol{r}\left(t_{f}, s\right)=\boldsymbol{R}_{f}^{\prime}(s)} \mathcal{D} \boldsymbol{r}(t, s) \delta\left(\boldsymbol{r}^{2}(t, s)-1\right) \prod_{l=1}^{2 \sigma} \int_{0}^{t_{f}} d t_{l} \int_{0}^{L} d s_{l} k_{a_{l}}\left(t_{l}, s_{l}\right) r_{a_{l}}\left(t_{l}, s_{l}\right)
$$


In the above equation the sum over repeated spatial indexes $a_{1}, \ldots, a_{2 \sigma}=1,2,3$ is understood. Due to the presence of the functional delta function, it is difficult to apply the Wick theorem in order to integrate over the field $\boldsymbol{r}(t, s)$. However, a strategy to evaluate $I_{2 \sigma}$ becomes clear after the discretization of both variables $t$ and $s$. Calling $I_{2 \sigma}\{N, \mathcal{M}, a, b\}$ the discretized version of $I_{2 \sigma}$, we obtain:

$$
\begin{aligned}
I_{2 \sigma}\{N, \mathcal{M}, a, b\} & =\prod_{n=1}^{N} \prod_{m=2}^{\mathcal{M}-1} \int d \boldsymbol{r}_{n m} \delta\left(\boldsymbol{r}_{n m}^{2}-1\right) \prod_{l=1}^{2 \sigma}\left(\sum_{n_{l}=1}^{N} a \sum_{m_{l}=2}^{\mathcal{M}-1} b k_{a_{l}, n_{l} m_{l}} r_{a_{l}, n_{l} m_{l}}\right) \\
& \times \prod_{n=1}^{N} \int d \boldsymbol{r}_{n 1} d \boldsymbol{r}_{n \mathcal{M}} A_{1} A_{\mathcal{M}}
\end{aligned}
$$

with the boundary conditions at the initial and final times fixed by Dirac delta functions inside the factors $A_{1}$ and $A_{\mathcal{M}}$ :

$$
A_{1}=\prod_{n=2}^{N} \delta\left(\boldsymbol{r}_{n 1}-\frac{\left(\boldsymbol{R}_{0, n}-\boldsymbol{R}_{0, n-1}\right)}{a}\right) \quad \text { and } \quad A_{\mathcal{M}}=\prod_{n=2}^{N} \delta\left(\boldsymbol{r}_{n \mathcal{M}}-\frac{\left(\boldsymbol{R}_{f, n}-\boldsymbol{R}_{f, n-1}\right)}{a}\right)
$$

Let us note that, due to the choice of Dirichlet boundary condition for $\boldsymbol{k}(t, s)$, the boundary values of this field are all zero, i. e. $\boldsymbol{k}_{n 1}=\boldsymbol{k}_{n \mathcal{M}}=0$ for $n=1, \ldots, N$. For this reason, the sums over the indices $m_{l}$ in Eq. (50) have been restricted in the range $2 \leq m_{l} \leq \mathcal{M}-1$ for $l=1, \ldots, 2 \sigma$. As a consequence, the variables at the boundary $\boldsymbol{r}_{n 1}$ and $\boldsymbol{r}_{n \mathcal{M}}$ in Eq. (50) are not present in the product $\prod_{l=1}^{2 \sigma}\left(\sum_{n_{l}=1}^{N} a \sum_{m_{l}=2}^{\mathcal{M}-1} b k_{a_{l}, n_{l} m_{l}} r_{a_{l}, n_{l} m_{l}}\right)$. They enter only in the terms $A_{1}$ and $A_{\mathcal{M}}$ and are not mixed with the other degrees of freedom to be integrated. Thanks to this fact, the integrals over $\boldsymbol{r}_{n 1}$ and $\boldsymbol{r}_{n \mathcal{M}}$ for $n=1, \ldots, N$ can be easily factored out from the expression of $I_{2 \sigma}\{N, \mathcal{M}, a, b\}$. The calculation of $I_{2 \sigma}\{N, \mathcal{M}, a, b\}$ requires the integration over the remaining $3 N(\mathcal{M}-2)$ variables $\boldsymbol{r}_{n m}$ for $1 \leq n \leq N$ and $2 \leq m \leq \mathcal{M}-1$ with the measure $d \boldsymbol{r}_{n m} \delta\left(\boldsymbol{r}_{n m}^{2}-1\right)$. The integrand consists in a sum of terms in which $2 \sigma$ components $r_{a_{l}, n_{l} m_{l}}$ of the vectors $\boldsymbol{r}_{n_{l} m_{l}}$ with various combinations of the indexes $a_{l}, m_{l}, n_{l}$ are multiplied together. Each of such components may appear inside the product with powers of any order comprised between 0 and $2 \sigma$. Clearly, only the integration over terms in which the indexes $a_{l}$ of the components and the discrete indexes $n_{l}, m_{l}$ are present in even combinations will not vanish identically. Basing ourselves on these observation, it is possible to establish the following rules in order to evaluate $I_{2 \sigma}\{N, \mathcal{M}, a, b\}$ :

1. Rearrange the terms appearing in $I_{2 \sigma}\{N, \mathcal{M}, a, b\}$ in such a way that the products of the $2 \sigma$ components $r_{a_{l}, n_{l} m_{l}}$ 's will be grouped into products of $2 l$-plets for $l=1, \ldots, \sigma$ 
characterized by the fact that inside each multiplet all the components have the same indices $n_{l}$ and $m_{l}$.

2. Eliminate the spatial indices $a_{l}$ by using the formula

$$
\sum_{i_{l}=1}^{3} k_{a_{l}, n_{l} m_{l}} r_{a_{l}, n_{l} m_{l}}=\left|\boldsymbol{k}_{n_{l} m_{l}}\right|\left|\boldsymbol{r}_{n_{l} m_{l}}\right| \cos \theta_{n_{l} m_{l}}
$$

3. Use the fact that, due to the delta functions $\delta\left(\boldsymbol{r}_{n m}^{2}-1\right)$ it is possible to put $\left|\boldsymbol{r}_{n_{l} m_{l}}\right|=1$ and integrate over the angles $\theta_{n_{l} m_{l}}$ using the formula:

$$
\int_{0}^{\pi} d \theta_{m n} \sin \theta_{n m} \cos ^{2 i} \theta_{n m}=2 \frac{1}{2 i+1} \quad i=0,1, \ldots
$$

Let $K_{l}$ be the number of $2 l$-plets mentioned in 1. Clearly, the possible values of $K_{l}$ are limited by the condition $\sum_{l=1}^{\sigma} 2 l K_{l}=2 \sigma$. This implies for instance that $K_{l}=1$ for $l>\sigma$. Moreover, the number of combinations for dividing $2 \sigma$ objects in a number $K_{1}$ of pairs, $K_{2}$ of 4 -plets etc. is given by:

$$
K=\frac{2 \sigma !}{2 K_{1} ! 4 K_{2} ! \cdots 2(\sigma-1) K_{\sigma-1} !}
$$

Following steps 1, and 2, of the above prescription, we may write:

$$
\begin{aligned}
& I_{2 \sigma}\{N, \mathcal{M}, a, b\}=\sum_{\substack{\sum_{1} K_{1}, \ldots, K_{\sigma} \\
2 l K_{l}=2 \sigma}} \frac{2 \sigma !}{2 K_{1} ! 4 K_{2} ! \cdots 2(\sigma-1) K_{\sigma-1} !} \prod_{l=1}^{\sigma} \sum_{n_{l, 1}, \ldots, n_{l, K_{l}}=1}^{N} a^{K_{l}} \sum_{m_{l, 1}, \ldots, m_{l, K_{l}}=1}^{\mathcal{M}} b^{K_{l}} \\
& \times\left|\boldsymbol{k}_{n_{l, 1} m_{l, 1}}\right|^{2 l} \ldots\left|\boldsymbol{k}_{n_{l, K_{l}} m_{l, K_{l}}}\right|^{2 l} \prod_{n, m=1}^{N, \mathcal{M}} \int_{0}^{+\infty} d\left|\boldsymbol{r}_{n m}\right| \delta\left(\left|\boldsymbol{r}_{n m}\right|^{2}-1\right)\left|\boldsymbol{r}_{n_{l, 1} m_{l, 1}}\right|^{2 l} \ldots\left|\boldsymbol{r}_{n_{l, K_{l}} m_{l, K_{l}}}\right|^{2 l} \\
& \times \int_{0}^{2 \pi} d \phi_{n m} \int_{0}^{\pi} d \theta_{n m} \sin \theta_{n m} \cos ^{2 l} \theta_{n_{l, 1} m_{l, 1}} \ldots \cos ^{2 l} \theta_{n_{l, K_{l}} m_{l, K_{l}}} A_{1} A_{\mathcal{M}}
\end{aligned}
$$

It is now possible to perform the remaining integrations according to prescription 3 , The result is:

$$
\begin{aligned}
& I_{2 \sigma}\{N, \mathcal{M}, a, b\}=Z_{2}[0 ; N, \mathcal{M}, a, b] \sum_{\substack{\sum_{l=1}^{\sigma} 2, \ldots, K_{\sigma} \\
2 l K_{l}=2 \sigma}} \frac{2 \sigma !}{2 K_{1} ! 4 K_{2} ! \cdots 2(\sigma-1) K_{\sigma-1} !} \\
& \times \prod_{l=1}^{\sigma} \sum_{n_{l, 1}, \ldots, n_{l, K_{l}}=1}^{N} a^{K_{l}} \sum_{m_{l, 1}, \ldots, m_{l, K_{l}}=1}^{\mathcal{M}} b^{K_{l}}\left|\boldsymbol{k}_{n_{l, 1} m_{l, 1}}\right|^{2 l} \ldots\left|\boldsymbol{k}_{n_{l, K_{l}} m_{l, K_{l}}}\right|^{2 l} \frac{1}{(2 l+1)^{K_{l}}}
\end{aligned}
$$




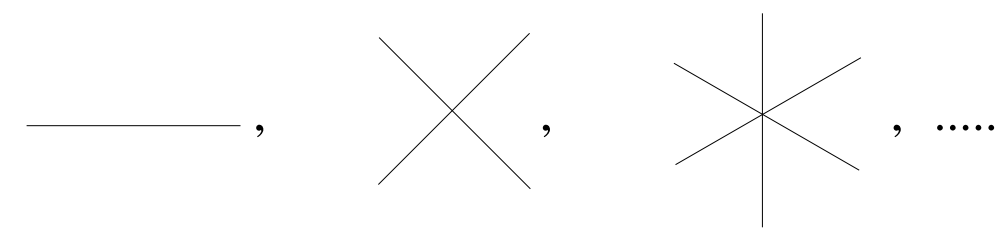

FIG. 1: This figure shows the Feynman diagrams corresponding to the terms $\int_{0}^{t_{f}} d t \int_{0}^{L} d s|\boldsymbol{k}(t, s)|^{2}$, $\int_{0}^{t_{f}} d t \int_{0}^{L} d s|\boldsymbol{k}(t, s)|^{4}, \int_{0}^{t_{f}} d t \int_{0}^{L} d s|\boldsymbol{k}(t, s)|^{6}, \ldots$ appearing in Eq. (58).

where

$$
Z_{2}[0 ; N, \mathcal{M}, a, b]=\prod_{n, m=1}^{N, \mathcal{M}} \int_{0}^{+\infty} d\left|\boldsymbol{r}_{n m}\right| \delta\left(\left|\boldsymbol{r}_{n m}\right|^{2}-1\right) \mid \int_{0}^{2 \pi} d \phi_{n m} \int_{0}^{\pi} d \theta_{n m} \sin \theta_{n m} A_{1} A_{\mathcal{M}}
$$

is the discrete version of $\mathcal{Z}_{2}[\boldsymbol{k}=0]$. The continuous limit of $I_{2 \sigma}\{N, \mathcal{M}, a, b\}$ starting from Eq. (56) gives:

$$
\begin{gathered}
I_{2 \sigma}=\mathcal{Z}_{2}[0] \sum_{\substack{K_{1}, \ldots \ldots, K_{\sigma} \\
\sum_{l=1}^{\sigma} 2 K_{l}=2 \sigma}} \frac{2 \sigma !}{2 K_{1} ! 4 K_{2} ! \cdots 2(\sigma-1) K_{\sigma-1} !} \\
\times \prod_{l=1}^{\sigma} \frac{1}{(2 l+1)^{K_{l}}} \int_{0}^{t_{f}} d t_{l, 1} \int_{0}^{L} d s_{l, 1}\left|\boldsymbol{k}\left(t_{l, 1}, s_{l, 1}\right)\right|^{2 l} \cdots \int_{0}^{t_{f}} d t_{l, K_{l}} \int_{0}^{L} d s_{l, K_{l}}\left|\boldsymbol{k}\left(t_{l, K_{l}}, s_{l, K_{l}}\right)\right|^{2 l}
\end{gathered}
$$

with

$$
\mathcal{Z}_{2}[0]=\lim _{\substack{a \rightarrow 0, N \rightarrow+\infty, N a=L \\ b \rightarrow 0, \mathcal{M} \rightarrow+\infty, \mathcal{M} b=t_{f}}} Z_{2}[0 ; N, \mathcal{M}, a, b]
$$

$\mathcal{Z}_{2}[0]$ contains the dependence on the boundary values of the fields $\boldsymbol{R}(t, s)$. Finally, substituting Eq. (58) in Eq. (48), we obtain the explicit expression of $\mathcal{Z}_{2}[\boldsymbol{k}]$. Having derived the functionals $\mathcal{Z}_{1}[\boldsymbol{k}, \boldsymbol{J}]$ and $\mathcal{Z}_{2}[\boldsymbol{k}]$, we may compute the full generating functional $\mathcal{Z}[\boldsymbol{J}]$ of Eq. (36):

$$
\begin{aligned}
\mathcal{Z}[\boldsymbol{J}] & =\mathcal{Z}_{2}[0] \int \mathcal{D} \boldsymbol{k}(t, s) e^{-S_{c l}(\boldsymbol{k}, \boldsymbol{J})} e^{-\frac{1}{4} \int_{0}^{t_{f}} d t d t^{\prime} \int_{0}^{L} d s G\left(t, t^{\prime}\right)\left(\boldsymbol{J}^{\prime}(t, s)+\boldsymbol{k}^{\prime}(t, s)\right) \cdot\left(\boldsymbol{J}^{\prime}\left(t^{\prime}, s\right)+\boldsymbol{k}^{\prime}\left(t^{\prime}, s\right)\right)} \\
& \times \sum_{\sigma=0}^{+\infty} \frac{(-\imath)^{2 \sigma}}{2 \sigma !} \sum_{\substack{K_{1}, \ldots, K_{\sigma} \\
\sum_{l=1}^{\sigma} 2 l K_{l}=2 \sigma}} \frac{2 \sigma !}{2 K_{1} ! 4 K_{2} ! \cdots 2(\sigma-1) K_{\sigma-1} !} \prod_{l=1}^{\sigma} \frac{1}{(2 l+1)^{K_{l}}} \\
& \times \int_{0}^{t_{f}} d t_{l, 1} \int_{0}^{L} d s_{l, 1}\left|\boldsymbol{k}\left(t_{l, 1}, s_{l, 1}\right)\right|^{2 l} \cdots \int_{0}^{t_{f}} d t_{l, K_{l}} \int_{0}^{L} d s_{l, K_{l}}\left|\boldsymbol{k}\left(t_{l, K_{l}}, s_{l, K_{l}}\right)\right|^{2 l}
\end{aligned}
$$

and perform the remaining integrations over the fields $\boldsymbol{k}(t, s)$. Let us note that the action in Eq. (60) is gaussian. The classical sector present in the term $S_{c l}(\boldsymbol{k}, \boldsymbol{J})$ of Eq. (42) introduces a coupling of $\boldsymbol{k}(t, s)$ with an external current which is proportional to the classical 
conformation $\boldsymbol{R}_{c l}(t, s)$. It is actually possible to rewrite the generating functional $\mathcal{Z}[\boldsymbol{J}]$ in a much more compact form by computing the following functional:

$$
\begin{aligned}
\mathcal{Z}[\boldsymbol{J}, \boldsymbol{\mu}] & =\exp \left\{-\int_{0}^{t_{f}} d t \int_{0}^{L} d s c \dot{\boldsymbol{R}}_{c l}^{2}(t, s)\right\} \\
& \times \exp \left\{-\imath \int_{0}^{t_{f}} d t d t^{\prime} \int_{0}^{L} d s G\left(t, t^{\prime}\right)\left(\boldsymbol{J}^{\prime}(t, s)+\boldsymbol{k}^{\prime}(t, s)\right) \cdot\left(\boldsymbol{J}^{\prime}\left(t^{\prime}, s\right)+\boldsymbol{k}^{\prime}\left(t^{\prime}, s\right)\right)\right\} \\
& \times \exp \left\{-\imath \int_{0}^{t_{f}} d t \int_{0}^{L} d s \boldsymbol{\mu}(t, s) \cdot \boldsymbol{k}(t, s)\right\}
\end{aligned}
$$

where $\boldsymbol{\mu}(t, s)$ is the external current that is needed to generate the correlation functions of the field $\boldsymbol{k}(t, s) . \mathcal{Z}[\boldsymbol{J}, \boldsymbol{\mu}]$ is the double generating functional of the correlation functions of the physical fields $\boldsymbol{R}(t, s)$ and of the auxiliary fields $\boldsymbol{k}(t, s)$. Knowing $\mathcal{Z}[\boldsymbol{J}, \boldsymbol{\mu}]$, the generating functional $\mathcal{Z}[\boldsymbol{J}]$ may be expanded as follows:

$$
\begin{aligned}
\mathcal{Z}[\boldsymbol{J}] & =\sum_{\sigma=0}^{+\infty} \sum_{\substack{K_{1}, \ldots, K_{\sigma} \\
\sum_{l=1}^{\sigma} 2 l K_{l}=2 \sigma}} \prod_{l=1}^{\sigma} \frac{1}{\left(2 l K_{l}\right) !} \frac{1}{(2 l+1)^{K_{l}}} \\
& \times\left.\left(\frac{\delta^{2}}{\delta \boldsymbol{\mu}\left(t_{l, 1}, s_{l, 1}\right) \cdot \delta \boldsymbol{\mu}\left(t_{l, 1}, s_{l, 1}\right)}\right)^{l} \cdots\left(\frac{\delta^{2}}{\delta \boldsymbol{\mu}\left(t_{l, K_{l}}, s_{l, K_{l}}\right) \cdot \delta \boldsymbol{\mu}\left(t_{l, K_{l}}, s_{l, K_{l}}\right)}\right)^{l} \mathcal{Z}[\boldsymbol{J}, \boldsymbol{\mu}]\right|_{\boldsymbol{\mu}=0}
\end{aligned}
$$

$\mathcal{Z}[\boldsymbol{J}, \boldsymbol{\mu}]$ can be computed in closed form. Its expression is given below:

$$
\begin{aligned}
\mathcal{Z}[\boldsymbol{J}, \boldsymbol{\mu}] & =\mathcal{Z}_{2}[0] \exp \left\{-\int_{0}^{t_{f}} d t \int_{0}^{L} d s\left(c \dot{\boldsymbol{R}}_{c l}^{2}+\imath \boldsymbol{J}^{\prime} \cdot \boldsymbol{R}_{c l}-\imath \boldsymbol{\mu} \cdot \boldsymbol{J}\right)\right\} \\
& \times \exp \left\{-\int_{0}^{t_{f}} d t d t^{\prime} \int_{0}^{L} d s d s^{\prime} \mathcal{G}\left(t, s ; t^{\prime}, s^{\prime}\right) \tilde{\boldsymbol{\mu}}(t, s) \cdot \tilde{\boldsymbol{\mu}}\left(t^{\prime}, s^{\prime}\right)\right\}
\end{aligned}
$$

where

$$
\mathcal{G}\left(t, s ; t^{\prime}, s^{\prime}\right)=\frac{c}{2} \frac{\partial^{2} \delta\left(t-t^{\prime}\right)}{\partial t^{\prime 2}} \sum_{n=1}^{+\infty} \frac{L^{2}}{(2 \pi n)^{2}} \sin \frac{2 \pi n s}{L} \sin \frac{2 \pi n s^{\prime}}{L}
$$

and

$$
\tilde{\boldsymbol{\mu}}=\boldsymbol{\mu}+\boldsymbol{R}_{c l}
$$

It is straightforward to realize that at each order in the index $\sigma$, the series in Eq. (62) contains products of vertices with $2 l$ external legs like those shown in Fig. 1 for $l=0,1,2, \ldots, \sigma$. The legs of the $2 l$-vertices are contracted in all possible ways in Eqs. (60) or (62) using the propagator (64). Convergence is granted for small values of the constant $c$. It is important to stress that the term $\mathcal{Z}_{2}[0]$, which contains a nontrivial dependence on the boundary conformations of $\boldsymbol{R}(t, s)$, appears in Eq. (63) as an overall factor that can be easily eliminated by choosing the normalization of the correlation functions. 


\section{A BRIEF DIGRESSION ON THE NONLINEAR SIGMA MODEL}

In this Section we will apply the method illustrated in the case of the inextensible chain to a two dimensional nonlinear sigma model with action:

$$
S_{s m}[J]=\int_{M} d^{2} x\left[\frac{g}{2}\left(\partial_{i} \boldsymbol{\phi}\right)^{2}+\boldsymbol{J} \boldsymbol{\phi}\right]
$$

where $\partial_{i}=\frac{\partial}{\partial x_{i}}, x=\left(x_{1}, x_{2}\right)$ and $M$ is a two dimensional manifold with Euclidean signature. To avoid complications with the choice of boundary conditions, $M$ is chosen to be a torus, so that periodic boundary conditions should be implemented for $x_{1}$ and $x_{2}$. The vector field $\phi(x)$ is subjected to the constraint:

$$
\phi^{2}(x)=1
$$

The generating functional of the nonlinear sigma model in path integral form looks very similar to that of the inextensible chain:

$$
Z_{s m}[J]=\int \mathcal{D} \phi e^{-S_{s m}[J]} \delta\left(\phi^{2}-1\right)
$$

Introducing the new fields $\boldsymbol{r}(x)$ and $\boldsymbol{k}(x)$, the integration over the physical field $\boldsymbol{\phi}$ can be performed without worrying about the functional Dirac delta function $\delta\left(\phi^{2}-1\right)$. The result of this integration is:

$$
\mathcal{Z}_{\text {sm }}[J]=\int \mathcal{D} \boldsymbol{k}(x) e^{-\frac{1}{4} \int_{M} d^{2} x d^{2} y G(x, y)(\boldsymbol{J}(x)+\boldsymbol{k}(x)) \cdot(\boldsymbol{J}(x)+\boldsymbol{k}(x))} \mathcal{Z}_{2}[k]
$$

Apart from the different topology of the underlying manifold $M, \mathcal{Z}_{2}[k]$ is the same as the functional $\mathcal{Z}_{2}[\boldsymbol{k}]$ given in Eq. (38):

$$
\mathcal{Z}_{2}[k]=\int \mathcal{D} \boldsymbol{r}(x) e^{\imath \int_{M} \boldsymbol{k}(x) \cdot \boldsymbol{r}(x)} \delta\left(\boldsymbol{r}^{2}(x)-1\right)
$$

One may thus evaluate this functional exactly as in the previous Section. The result is an expansion of $\mathcal{Z}_{2}[k]$ at all orders in powers of $\boldsymbol{k}(x)$ which converges for small values of the coupling constant $g$. The most relevant quantity to be computed in order to derive term by term at all orders the contributions to this expansion is the extended generating functional $\mathcal{Z}_{s m}[J, \mu]$ analogous to that of Eq. (61). After a long but straightforward calculation, one obtains apart from a trivial overall factor the following expression of $\mathcal{Z}_{s m}[J, \mu]$ :

$$
\mathcal{Z}_{s m}[J, \mu]=e^{\imath \int_{M} d^{2} x \boldsymbol{J} \cdot \boldsymbol{\mu}} e^{-\int_{M} d^{2} x d^{2} y \mathcal{G}(x, y) \boldsymbol{\mu}(x) \cdot \boldsymbol{\mu}(y)}
$$


where

$$
\mathcal{G}(x, y)=\frac{g}{2} \Delta_{x} \delta^{2}(x, y)
$$

and $\Delta_{x}=\frac{\partial^{2}}{\partial x_{1}^{2}}+\frac{\partial^{2}}{\partial x_{2}^{2}}$.

\section{CONCLUDING REMARKS}

In this work a recipe has been presented for constructing the generating functional of constrained stochastic systems with an arbitrary number of degrees of freedom and general constraints. The main idea is that the variables to be constrained are allowed to fluctuate thanks to the addition of the auxiliary noises $\tilde{\nu}_{\alpha}$ and of the fictitious variables $\xi_{\alpha}$, $\alpha=1, \ldots, M$. In this way, the conditions $C_{\alpha}(\{\boldsymbol{R}\})$ in Eq. (13) are not fixed exactly, but are subjected to stochastic fluctuations. The original constraints (1) are recovered in the "rigid" limit $\eta_{\alpha}, \tilde{D}_{\alpha} \rightarrow 0$. The set of equations (12) and (13) which describes the dynamics of both physical and fictitious degrees of freedom is formally a system of overdamped Langevin equations. Starting from a system of that kind it is possible to derive a path integral expression of the related generating functional by using the standard techniques valid for stochastic systems without constraints. In the rigid limit $\eta_{\alpha}, \tilde{D}_{\alpha}=0$, the generating functional $Z[\boldsymbol{J}]$ is given in Eq. (24). Remarkably, with this procedure the jacobian determinant appearing after the change of variables $\boldsymbol{\nu}_{i}, \tilde{\nu}_{\alpha} \longrightarrow \boldsymbol{R}_{i}, \xi_{\alpha}$ remains relatively simple. For comparison, the standard treatment of constrained path integrals, see for instance [36], produces complicated jacobian determinants of block matrices. The path integral expressions obtained in this way are very useful at a theoretical level, because they allow for example to check if the Parisi-Wu quantization scheme delivers in the equilibrium limit the desired generating functional of the theory to be quantized [8, 9], but are very cumbersome in concrete calculations of physical observables. The method discussed in this work produces instead relatively simple generating functionals. In the case of the inextensible chain in the absence of external interactions, we obtain for instance the GNL $\sigma \mathrm{M}$ of Ref. [31], which allows the computation of several measurable quantities. For example, the dynamical form factor of the chain has been evaluated in [44]. Always in [44] it has been estimated how the fluctuations of the distance between two arbitrary points on the chain are influenced by physical parameters like the length of the chain and the relaxation time $\tau$. The novelty of the present approach with respect to [31] is the possibility to add also the external forces $\boldsymbol{f}_{i}$. 
Of course, the expressions of the generating functionals obtained here are still complicated due to the presence of the Dirac delta functions that are needed to impose the constraints. For this reason, in this work a technique is developed for computing the the generating functional of two dimensional field theories in the presence of a Dirac delta functions like that appearing in the GNL $\sigma \mathrm{M}$ of Eq. (29). Thanks to the introduction of the auxiliary field $\boldsymbol{r}(t, s)$, the difficulties related to the delta function are confined to the computation of the path integral $\mathcal{Z}_{2}[\boldsymbol{k}]$ of Eq. (38). Apart from the boundaries at $t=0$ and $t=t_{f}$, Eq. (46) shows that $\mathcal{Z}_{2}[\boldsymbol{k}]$ formally consists of the infinite product over the time index $m$ of terms that are nothing but the Fourier transform of the probability function of a freely jointed chain in statistical mechanics. The functional $\mathcal{Z}_{2}[\boldsymbol{k}]$ has been evaluated here in the form of a series converging for small values of the parameter $c$ given of Eq. (30). Despite the fact that Eq. (62) looks like a perturbative expansion, all terms entering in the expansion can be computed in closed form. Indeed, the main ingredient in Eq. (62) is the extended generating functional $\mathcal{Z}[\boldsymbol{J}, \boldsymbol{\mu}]$, which generates both correlation functions of the physical fields $\boldsymbol{R}(t, s)$ and of the auxiliary fields $\boldsymbol{k}(t, s)$. The exact expression of $\mathcal{Z}[\boldsymbol{J}, \boldsymbol{\mu}]$ is given by Eq.(63).

Finally, the techniques presented in this work can be extended to other systems with constraints. For instance, we have seen there are many similarities between the two dimensional nonlinear sigma model and the GNL $\sigma \mathrm{M}$. Despite the initial difference of the constraints, after the introduction of auxiliary fields the generating functional of both theories may be computed in the same way. In particular, the extended generating functional $\mathcal{Z}_{s m}[J, \mu]$ of the correlation functions of the physical fields $\boldsymbol{\phi}(x)$ and the auxiliary fields $\boldsymbol{k}(x)$ for the nonlinear sigma model has been exactly derived in Eq. (71). We hope that in the future it will be possible to apply these methods also to the statistical mechanics of polymer interactions in the presence of topological entanglement [45 47]. In statistical mechanics, in fact, the probability function of a system of long and flexible polymer chains obeys pseudoSchrödinger equations that, for certain aspects, are similar to those of Fokker-Planck. A nice physical application could be the study of the influence of topological constraints on the interactions in colloid-polymer mixtures [48, 49]. 


\section{ACKNOWLEDGMENTS}

Support from the National Science Foundation of the United States under Grant No. NSF PHY05-51164 and by the Polish National Center of Science, scientific project No. N N202 326240 is gratefully acknowledged.

[1] C. Bustamante, J. F. Marko, E. D. Siggia and S. Smith, Science 265 (1994), 415.

[2] C. Storm and P. C. Nelson, Phys. Rev. E 67 (2003), 051906.

[3] J. F. Marko and E. D. Siggia, Macrom. 28 (1995), 8759.

[4] T. B. Liverpool, Phys. Rev. E 72 (2005), 021805.

[5] M. Febbo, A. Milchev, V. G. Rostiashvili, D. Dimitrov and T. A. Vilgis, Jour. Chem. Phys. 129 (15) (2008), 154908.

[6] W. Tomaszewski and P. Pieranski, New Jour. Phys. 7 (2005), 45.

[7] K. Ward, F.Bertails, T.-Y. Kim, S. R. Marschner, M.-P. Cani and M. Lin, IEEE Transactions on Visualization and Computer Graphics (TVCG), 13 (2) (2007), 213.

[8] R. Mochizuki, Prog. Theor. Phys. 85 (1991), 407.

[9] M. Namiki, I. Ohba and K. Okano, Prog. Theor. Phys. 72 (1984), 350.

[10] J. Zinn - Justin, Quantum Field Theory and Critical Phenomena, Clarendon Press, Oxford, 2002.

[11] D. Hochberg, C. Molina-Paris, J.-P. Mercader and M. Visser, Phys. Rev. E 60 (1999), 6343.

[12] P. H. Damgaard and H. Hüffel, Phys. Rep. 152 (1987), 227.

[13] P. C. Martin, E. D. Siggia and H. A. Rose, Phys. Rev. A 8 (1973), 423.

[14] M. Doi and S.F. Edwards, The Theory of Polymer Dynamics (Clarendon Press, Oxford, 1986).

[15] M. Fixman, Proc. Nat. Acad. Sci. USA 71 (8) (1974), 3050.

[16] R. Granek, J. Phys. II France 7 (1997), 1761.

[17] J. R. Blundell and E. M. Terentjev, Jour. Phys. A: Math. Gen. 40 (2007), 10951.

[18] L. C. Gomes and R. Lobo, Rev. Bras. Fis. 9 (1979), 797.

[19] D. P. Petera and M. Muthukumar, Jour. Chem. Phys. 111 (1999), 7614.

[20] E. J. Hinch, Jour. Fluid Mech. 271 (1994), 219.

[21] C. F. Curtiss and R. Byron Bird, Adv. Pol. Science 125, (Springer Verlag, Berling, Heidelberg, 
1996).

[22] T. B. Liverpool and A. C. Maggs, Macromol. 34 (2001), 6064.

[23] P. S. Doyle and P. T. Underhill, Numerical Brownian Dynamics Simulations of Polymers and Soft Matter, published in handbook of Materials Modeling, (Springer Netherland, 2005), 2619.

[24] R. Everaers, Eur. Phys. Jour. B 4 (1998), 341.

[25] F. F. Abraham, Adv. Phys. 35 (10) (1986), 1.

[26] A. Montesi, D. C. Morse and M. Pasquali, Jour. Chem. Phys. 122 (2005), 084903.

[27] E. Klaveness and A. Elgsaeter, Jour. Chem. Phys. 110 (23) (1999), 11608.

[28] J. E. Butler and E. S. G. Shaqfeh, Jour. Chem. Phys. 122 (2005), 014901.

[29] P. Echenique, I. Calvo and J. L. Alonso, Jour. Comput. Chem. 27 (14) (2006), 1733.

[30] E. A. J. F. Peters, Polymers in flow, modelling and simulating, (Ponsen \& Loojen, The Netherlands 2000), ISBN 90-370-0183-1.

[31] F. Ferrari, J. Paturej and T. A. Vilgis, Phys. Rev. E, 77, 021802, 2008.

[32] F. Ferrari and J. Paturej, Jour. Phys. A: Mathematical and Theoretical 42 (14) (2009), 145002.

[33] F. Ferrari and M. Pyrka, Dynamical aspects of inextensible chains, Preprint no. NSF-KITP11-092, arXiv:1101.5780v2 [cond-mat.stat-mech].

[34] J. R. Klauder, Ann. Phys. 254 (1997), 419.

[35] E. Gozzi, M. Reuter and W. D. Thacker, Phys. Rev. D 40 (1989), 3363; ibid. 46 (1992), 757.

[36] C. Itzykson and J. B. Zuber, Gauge field theory, (McGraw-Hill, New York, 1980).

[37] S. F. Edwards and A. G. Goodyear, J. Phys. A: Gen. Phys. 5 (1972), 965.

[38] S. F. Edwards and A. G. Goodyear, J. Phys. A: Gen. Phys. 5 (1972), 1188.

[39] S. F. Edwards and A. G. Goodyear, J. Phys. A: Gen. Phys. 6 (1973), L31.

[40] M. Gell-Mann and M. Levy, Nuovo Cim. 16 (1960), 705.

[41] M. Chaichian and A. Demichev, Path Integrals in Physics, Vol. 1, (IOP Publishing, 2001).

[42] S. A. Rice and H. L. Frisch, Ann. Rev. Phys. Chem. 11 (1960), 187.

[43] H. Kleinert, Path Integrals in Quantum Mechanics, Statistics, Polymer Physics, and Financial Markets, (World Scientific Publishing, 3nd Ed., Singapore, 2003).

[44] F. Ferrari, J. Paturej and T. A. Vilgis, Phys. Atomic Nuclei 73 (2) (2010), 295.

[45] A. L. Kholodenko and T. A. Vilgis, Phys. Rep. 298 (1998), 251.

[46] A. Vologodskii and V.V. Rybenkov, Phys. Chem. Chem. Phys. 11, 10543 (2009).

[47] J. F. Marko, Phys. Rev. E 79 (2009), 051905; Jour. Stat. Phys. 142 (2011), 1353. 
[48] A. I. Chervanyov and G. Heinrich, J. Chem. Phys. 131 (2009), 234907.

[49] A. L. Chervanyov and F. Ferrari, work in progress. 